\title{
COMPACT AND COMPACTLY GENERATED SUBGROUPS OF LOCALLY COMPACT GROUPS
}

\author{
R. W. BAGLEY, T. S. WU AND J. S. YANG
}

(Communicated by Jonathan M. Rosenberg)

\begin{abstract}
Our main interest is the existence of maximal compact normal subgroups of locally compact topological groups and its relation to compactly generated subgroups. If a topological group $G$ has a compact normal subgroup $K$ such that $G / K$ is a Lie group and every closed subgroup of $G$ is compactly generated, we call $G$ an $\mathscr{H}(c)$-group. If $G$ has a maximal compact normal subgroup $K$ such that $G / K$ is a Lie group, we call $G$ an $\mathscr{H}$-group. If $G$ is an $\mathscr{H}$.(c)-group, then $G$ is a hereditary $\mathscr{H}$-group in the sense that every closed subgroup is an $\mathscr{H}$-group. If $H$ is a closed normal subgroup of $G$ and both $H, G / H$ are $\mathscr{H}(c)$-groups, then $G$ is an $\mathscr{H}(c)$-group. A corollary of this is that a compactly generated solvable group whose characteristic open subgroups are compactly generated is an $\mathscr{H}$-group. If $G$ has a compactly generated closed normal subgroup $F$ such that both $F / F_{0} \quad G / F$ are $\mathscr{H}$-groups, then $G$ is an $\mathscr{H}$-group.
\end{abstract}

\section{INTRODUCTION}

In recent years there has been considerable interest in the existence of maximal compact, as well as compact normal, subgroups of locally compact topological groups. Compactly generated groups play a very special role in the existence of such subgroups. From results of G. Hochschild, D. Montgomery, L. Zippin, and G. D. Mostow $[9,10,11]$ we have the important basic result that, if $G / G_{0}$ is compact, then $G$ has a maximal compact normal subgroup with Lie factor, i.e., $G / K$ is a Lie group (See Lemma 1 [2]). S. Grosser and M. Moskowtiz, 3.20 [7], proved that a compactly generated $F C$-group has a maximal compact normal subgroup with Lie factor.

In [3] we called $G$ an $\mathscr{H}$-group if $G$ has a maximal compact normal subgroup with Lie factor. Here we continue the study begun in that paper. If every closed subgroup of a topological group $G$ is compactly generated and $G$ has some compact normal subgroup with Lie factor, we call $G$ an $\mathscr{H}(c)$-group. We first prove that an $\mathscr{H}(c)$-group is a heretidary $\mathscr{H}$-group in the sense that every closed subgroup is an $\mathscr{H}$-group, Lemma 1 . We use this and other lemmas to prove that $G$ is an $\mathscr{H}(c)$-group if $G$ has a closed normal subgroup $H$ such

Received by the editors December 9, 1988 and, in revised form, March 27, 1989.

1980 Mathematics Subject Classification (1985 Revision). Primary 22A05. 
that $H$ and $G / H$ are $\mathscr{H}(c)$-groups, Theorem 1. One of the corollaries of this theorem is that a compactly generated solvable group whose characteristic open subgroups are compactly generated is an $\mathscr{H}$-group.

In general a factor group of an $\mathscr{H}$-group is not an $\mathscr{H}$-group; and there are groups $G$ which are not $\mathscr{H}$-groups even though $G / H$ and $H$ are, where $H$ is a closed normal subgroup of $G$, Example 3 . We obtain results related to these situations. If $G$ has a compactly generated closed normal subgroup $F$ such that $G / F$ and $F / F_{0}$ are $\mathscr{H}$-groups, then $G$ is an $\mathscr{H}$-group, Theorem 2. If in Theorem 2 the condition that $F / F_{0}$ is an $\mathscr{H}$-group is relaxed to that of $F$ being an $\mathscr{H}$-group, then the same conclusion holds if $F$ is a $C A$-group and $B(F)$ is compactly generated. By $B(G)$ and $P(G)$ we denote the set of bounded elements and the set of periodic elements of $G$ respectively. Thus $B(G)$ is the set of elements of $G$ with relatively compact conjugacy classes and $P(G)$ consists of those elements which are contained in compact subgroups. For extensive work regarding these sets see $[14,17]$. It is easy to see that $B(G)$ is a characteristic subgroup but not necessarily closed. In Example 2, [5] $B(G)$ is not closed but is dense in $G$. If $G$ is an $\mathscr{H}$-group, then $B(G)$ and $P(G) \cap B(G)$ are closed subgroups, Proposition 1.5 [3].

Definition. A locally compact topological group $G$ is an $\mathscr{H}$-group if $G$ has a maximal compact normal subgroup $K$ with Lie factor, i.e. $G / K$ is a Lie group. The group $G$ is an $\mathscr{H}(c)$-group if $G$ has a compact normal subgroup with Lie factor and every closed subgroup of $G$ is compactly generated. $G$ is an $\mathscr{H}(d)$-group if $G$ has a compact normal subgroup with Lie factor and every subgroup of an arbitrary discrete factor of $G$ is finitely generated. A discrete factor of $G$ is a group $G / G^{\prime}$, where $G^{\prime}$ is an open normal subgroup of $G$.

It is easy to see that a locally compact group $G$ is an $\mathscr{H}(d)$-group if and only if $G$ has a compact normal subgroup $K$ such that $G / K$ is a Lie group and every subgroup of $G / K G_{0}$ is finitely generated. (Note that $K G_{0}$ is an open normal subgroup of $G$ by Theoren 1.3 [4].)

A closed subgroup of an $\mathscr{H}$-group is not necessarily an $\mathscr{H}$-group, Example 1. However, it is obvious that every closed subgroup of an $\mathscr{H}(c)$-group is an $\mathscr{H}(c)$-group.

Lemma 1. If $G$ is an $\mathscr{H}(c)$-group $\{\mathscr{H}(d)$-group $\}$, then $G$ is a hereditary $\mathscr{H}$ group $\{$ an $\mathscr{H}$-group $\}$.

Proof. The first reading follows from the second and the fact that the property of being an $\mathscr{H}(c)$-group is hereditary. To prove the second reading let $G^{\prime}$ be an open normal subgroup of $G$ such that $G^{\prime} / G_{0}$ is compact [1]. If $G / G^{\prime}$ has a maximal finite normal subgroup $F / G^{\prime}$, then $F / G_{0}$ is compact and the maximal compact normal subgroup $K$ of $F$ guaranteed by Lemma 1, [2] is maximal compact normal in $G$ with Lie factor, as desired. Thus, it is sufficient to prove that $G / G^{\prime}$ has a maximal finite normal subgroup. From this point we can assume $G$ is a discrete group each of whose normal subgroups is finitely generated. Now we apply Zorn's lemma to the collections of finite normal 
subgroups of $G$. Let $\left\{F_{i}\right\}$ be an ordered by inclusion increasing chain of finite normal subgroups of $G$. Let $F=\bigcup F_{i}$. Since $F$ is finitely generated, it follows that $F$ is the maximal element of $\left\{F_{i}\right\}$ and by Zorn's lemma $G$ has a maximal finite normal subgroup. This completes the proof.

Lemma 2. If $G$ has a discrete finitely generated normal subgroup $H$ whose center is also finitely generated, $G / H$ is compact and $H$ has a maximal finite normal subgroup, then $G$ is an $\mathscr{H}$-group.

Proof. We assume the maximal finite normal subgroup of $H$ is trivial. Let $F=Z_{G}(H)$. Then $F$ is open since $H$ is discrete and finitely generated. It follows that $F / F \cap H \cong F H / H$ is compact. Now $F$ is compactly generated since $F \cap H$ is in the center of $H$ and, by hypothesis, finitely generated. Thus $F$ is an $\mathscr{H}$-group by 4.5 [6]. Consequently $G$ is an $\mathscr{H}$-group.

Lemma 3. If $G$ has a closed normal subgroup $H$ which is an $\mathscr{H}(d)$-group and $G / H$ is compact, then $G$ is an $\mathscr{H}(d)$-group.

Proof. Let $H_{1}$ be the maximal compact normal subgroup of $H$ with Lie factor provided by Lemma 1 . Then $G$ is an $\mathscr{H}$-group if $G / H_{1}$ is. Also $G / H_{1}$ and $H / H_{1}$ satisfy the hypothesis on $G$ and $H$ of the lemma. Thus, we can assume that $H$ is a Lie group with no nontrivial compact normal subgroup. Every subgroup of $H / H_{0}$ is finitely generated by hypothesis. Now $G / H_{0}$ and $H / H_{0}$ satisfy the hypothesis of Lemma 2. Thus, $G^{\prime}=G / H_{0}$ is an $\mathscr{H}$-group. Let $K^{\prime}$ be the maximal compact normal subgroup of $G^{\prime}$. Let $N=\pi^{-1}\left(K^{\prime}\right)$ where $\pi: G \rightarrow G^{\prime}$ is the canonical homomorphism. Then $N$, being almost connected, has a maximal compact normal subgroup $K$ such that $N / K$ is a Lie group. It follows that $K$ is maximal compact normal in $G$. Since $N / K$ and $G / N \cong G^{\prime} / K^{\prime}$ are Lie, it follows that $G / K$ is a Lie group.

To prove that every subgroup of a discrete factor of $G$ is finitely generated we let $P$ be an open normal subgroup of $G$ and let $F / P$ be a subgroup of $G / P$. We have the following: $F H / H P \cong(F H / P) /(H P / P)=(F / P)(H P / P) /$ $(H P / P) \cong(F / P) /(F / P) \cap(H P / P)=(F / P) /((F \cap H P) / P)$. Thus $(F / P) /$ $((F \cap H P) / P)$ is finite since $F H / H P$ is. Also, $F \cap H P / P$ is finitely generated since it is a subgroup of $H P / P \cong H / H \cap P$, a discrete factor of the $\mathscr{H}(d)$ group $H$. It follows that $F / P$ is finitely generated, and the proof is complete.

Lemma 4. A locally compact group $G$ is an $\mathscr{H}(c)$-group if and only if $G$ is an $\mathscr{H}(d)$-group and every closed subgroup of $G_{0}$ is compactly generated.

Proof. The "only if" part is obvious. On the other hand suppose $G$ is an $\mathscr{H}(d)$ group. By Lemma 1 and the fact that $G$ is an $\mathscr{H}(c)$-group if $G / K$ is, where $K$ is compact normal in $G$, we can assume that $G$ is a Lie group. Now we let $F$ be any closed subgroup of $G$. Then $F G_{0} / G_{0} \cong F / F \cap G_{0}$ is finitely generated being a subgroup of the discrete factor $G / G_{0}$. By hypothesis $F \cap G_{0}$ is compactly generated; so $F$ is compactly generated as desired.

Lemma 5. If $G$ has a closed normal subgroup $H$ such that $G / H$ and $H$ are $\mathscr{H}(d)$-groups $\{\mathscr{H}(c)$-groups $\}$, then $G$ is an $\mathscr{H}(d)$-group $\{\mathscr{H}(c)$-group $\}$. 
Proof. For the first reading we use Lemma 1 to obtain a maximal compact normal subgroup $M / H$ of $G / H$ such that $(G / H) /(M / H) \cong G / M$ is a Lie group. By Lemmas 3 and 1, we have a maximal compact normal subgroup $K$ of $M$ such that $M / K$ is Lie. Thus $K$ is normal in $G$ and $G / K$ is Lie. Now let $P$ be an open normal subgroup of $G$ and let $F / P$ be a subgroup of the discrete factor $G / P$. We have $F H / H P \cong(F H / P) /(H P / P)=(F / P)(H P / P) /(H P / P) \cong$ $(F / P) /(F / P) \cap(H P / P)=(F / P) /((F \cap H P) / P)$. The last group is finitely generated since it is isomorphic to $F H / H P \cong(F H / H) /(H P / H)$, a subgroup of the discrete factor $(G / H) /(H P / H)$. It follows that $F / P$ is finitely generated since $(F \cap H P) / P$ is a subgroup of $H P / P \cong H / H \cap P$, a discrete factor of $H$. This completes the proof of the first reading.

From the proof above and Lemma 4, to prove the second reading of the lemma, it is sufficient to prove that every closed subgroup of $G_{0}$ is compactly generated; and without loss of generality we can assume $G$ is a connected Lie group. By Theorem 2.8 [4] it is sufficient to prove that $G$ has no noncompact simple factor.

Suppose $G$ contains a noncompact simple factor $S$, an analytic subgroup. Then $H \cap S$ is discrete. Thus, since $\mathscr{L}(S)=\mathscr{L}(S / H \cap S)$ we can assume that $S \cap H=e$. For $G / H \cap S$ and $H / H \cap S$ satisfy the hypothesis on $G$ and $H$; and $G / H \cap S$ has the noncompact simple factor $S / H \cap S$. Now $S \cong H S / H$, algebraically. Thus $\bar{S}=\overline{H S} / H$ identifying $S$ as a subgroup of $G / H$. From Proposition 1.4 [16] we have $\bar{S}=Z S \subset G / H$ where $Z$ is in the center of $\bar{S}$. Thus $A d S=A d \bar{S}$, II, 5.2, page 119 [8]. As in the proof of 2.8 [4] $A d(S)$ contains a closed subgroup $N$ which is not compactly generated. Thus $\operatorname{Ad}^{-1}(N) \subset \bar{S}$. This contradicts the fact that every closed subgroup of $G / H$ is compactly generated.

Theorem 1. If the locally compact group $G$ contains a sequence of closed normal subgroups $G=G_{1} \supset G_{2} \supset \cdots \supset G_{n}=e$ such that $G_{i} / G_{i+1}, i=1, \ldots, n-1$, is an $\mathscr{H}(d)$-group $\{\mathscr{H}(c)$-group $\}$ then $G$ is an $\mathscr{H}(d)$-group $\{\mathscr{H}(c)$-group $\}$.

Proof. This is an immediate consequence of Lemma 5.

Corollary 1. If a locally compact compactly generated solvable group $G$ has compactly generated derived subgroups, then $G$ is an $\mathscr{H}(c)$-group.

Proof. Each of the factor groups from the derived series is compactly generated Abelian.

Corollary 2. If a locally compact solvable group $G$ contains a sequence of closed normal subgroup $G=G_{1} \supset G_{2} \supset \cdots \supset G_{n}=e$, and every closed subgroup of $G_{i} / G_{i+1}$ is compactly generated, then $G$ is an $\mathscr{H}(c)$-group.

Proof. If $n=2$, then every closed subgroup of $G$ is compactly generated; so $G$ is an $\mathscr{H}(c)$-group. The corollary now follows by induction from the fact that, if $G=G_{1} \supset G_{2} \supset G_{3}=e$ and both of $G / G_{2}, G_{2}$ have every closed subgroup compactly generated, then $G$ is an $\mathscr{H}(c)$-group by Corollary 1 and Theorem 1 . 
Corollary 3. If $G$ is a compactly generated group, $G / G_{0}$ is solvable, and every characteristic open subgroup of $G$ is compactly generated, then $G$ is an $\mathscr{H}$-group and $G / G_{0}$ is an $\mathscr{H}(c)$-group.

Proof. It is sufficient to prove the corollary for totally disconnected groups. For, if $G$ satisfies the hypothesis so does $G / G_{0}$, and, if $G / G_{0}$ is an $\mathscr{H}$-group, then $G$ is an $\mathscr{H}$-group. Thus, we assume that $G$ is totally disconnected and not an $\mathscr{H}(c)$-group. By Corollary 1 we can choose $G^{\prime}$ to be the first subgroup in the derived sequence of $G$ which is not compact generated. It follows from that corollary that $G / G^{\prime}$ is an $\mathscr{H}(c)$-group. Let $N / G^{\prime}$ be the maximal compact open normal subgroup of $G / G^{\prime}$. Since $N / G^{\prime}$ is characteristic in $G / G^{\prime}$, it follows that $N$ is characteristic in $G$. Thus $N$ is compactly generated by hypothesis; so $G^{\prime}$ is compactly generated, a contradiction. (If $V$ is a compact neighborhood of the identity in $N$ which generates $N$ and $N=C G^{\prime}$ where $C$ is compact, then $V C \cap G^{\prime}$ generates $G^{\prime}$.) The proof is complete.

The following is an example of a locally compact group $G$ which is an $\mathscr{H}$ group but $G / G_{0}$ is not an $\mathscr{H}$-group. $G$ also has a closed subgroup which is not an $\mathscr{H}$-group.

Example 1. Let $G=R^{2} \times{ }_{\eta} D$ be the semidirect product of the discrete group $D=\left\{e^{2 \pi i t}: t=m / 2^{n}\right.$ where $m$ and $n$ are integers $\}$ and the plane $R^{2}$ with $D$ acting on $R^{2}$ by rotations. The maximal compact normal subgroup of the Lie group $G$ is the identity. Thus $G$ is an $\mathscr{H}$-group; but $G / R^{2} \cong D$ does not have a maximal finite subgroup. Thus the closed subgroup $D$ is not an $\mathscr{H}$-group.

This example is a subgroup of a group frequently cited [12]. It indicates that rather stringent conditions may be necessary to insure that the factor group of an $\mathscr{H}$-group is an $\mathscr{H}$-group. In this connection we have the following.

Proposition 1. If $G$ is an $\mathscr{H}$-group and $F$ is a compactly generated closed normal subgroup of $G$ such that $B(F)=F$ and $F / P(F)$ is discrete, then $G / F$ is an $\mathscr{H}$-group.

Proof. We can assume that the maximal compact normal subgroup of $G$ is trivial and that $G$ is a Lie group. By $1.8[14] F / P(F)$ is discrete, Abelian, and torsion free. Then, $F / P(F)$ is finitely generated and $P(F)$ is maximal compact normal in $F$, Theorem $5.5[6]$. Since $G / P(F)$ and $F / P(F)$ satisfy the hypothesis of the proposition and $G / F \cong(G / P(F)) /(F / P(F))$, we can assume that $P(F)$ is trivial. Thus, $F$ is a finitely generated Abelian torsion free group. Let $G_{1}$ be the group generated by the collection of all subgroups $H$ of $G$ such that $H / F$ is compact; and let $\rho: G_{1} \rightarrow \mathscr{A}(F)$ be the canonical map into the automorphism group of $F$ with the usual topology making it a topological group [16]. Thus $G_{1} / F=B(G / F) \cap P(G / F)$. Since $F \cong Z^{n}$, $\rho\left(G_{1}\right) \cong G_{1} / k(\rho)$ is finite where $k(\rho)$ is the kernel of $\rho$. Let $G_{2}$ be any normal subgroup of $G_{1}$ such that $F \subset G_{2} \subset k(\rho)$ and $G_{2} / F$ is compact. Thus $G_{2}$ is a $(Z)$-group and consequently has a maximal compact normal subgroup. Since this compact subgroup is normal in $G$ it must be trivial whence $G_{2} / F$ is 
trivial. Since the collection of all such subgroups $G_{2}$ geneerates $k(\rho)$, it follows that $k(\rho)=F$ and consequently $G_{1} / F$ is finite. Thus $B(G / F) \cap P(G / F)$ is finite and $G / F$ is an $\mathscr{H}$-group by Proposition 1.5 [3] as desired.

The center of a finitely generated group is not necessarily finitely generated, page $65[11]$. Thus the hypothesis that $B(F)$ is compactly generated in the proposition above is necessary.

A Lie group with maximal compact subgroups can have closed normal subgroups with no maximal compact subgroups [2]. In the following we obtain some pertinent results.

Lemma 6. Let $G$ be a locally compact group with maximal compact subgroups any two of which are conjugates, and suppose each compact subgroup of $G$ is contained in a maximal one. If $F$ is a closed normal Lie subgroup of $G$, then $F$ has maximal compact subgroups any two of which are conjugates by an element of $G$. The maximal compact subgroups of $F$ are those groups $K \cap F$ where $K$ is maximal compact in $G$.

Proof. Let $K$ be a maximal compact subgroup of $G$. Suppose $K \cap F$ is not maximal compact in $F$. Then there exists a maximal compact subgroup $K^{\prime}$ of $G$ such that $K^{\prime} \cap F$ properly contains $K \cap F$. Let $g \in G$ such that $g K^{\prime} g^{-1}=$ $K$. Now $g\left(K^{\prime} \cap F\right) g^{-1}=K \cap F \subset K^{\prime} \cap F$. Since $\left(K^{\prime} \cap F\right)_{0}$ is an open connected subgroup of $K^{\prime} \cap F$, we have $g\left(K^{\prime} \cap F\right)_{0} g^{-1}=\left(K^{\prime} \cap F\right)_{0}$. Since $K^{\prime} \cap F$ is compact it is the union of a finite number of cosets of $\left(K^{\prime} \cap F\right)_{0}$. It follows that $K^{\prime} \cap F=g\left(K^{\prime} \cap F\right) g^{-1}=K \cap F$ which contradicts the assumption that $K \cap F$ is not maximal in $F$. The remaining part of the conclusion is obvious.

Proposition 2. Let $G$ be a locally compact group with maximal compact subgroups any two of which are conjugates, and suppose each compact subgroup of $G$ is contained in a maximal one. If $F$ is a closed normal subgroup of $G$ and there is a compact subgroup $N$ of $F$ normal in $G$ such that $F / N$ is a Lie group, then $F$ contains maximal compact subgroups, those groups $K \cap F$ where $K$ is maximal compact in $G$. In addition $F$ is an $\mathscr{H}$-group.

Proof. It follows from Lemma 6 that $F / N$ has maximal compact subgroups of the form $(K / N) \cap(F / N)$ where $K$ is maximal compact in $G$. Thus the maximal compact subgroups $K$ of $G$ lead to the maximal compact subgroups $K \cap F$ in $F$. Now let $H$ be the intersection of all the maximal compact subgroups of $F$. Then $H$ is the maximal compact normal subgroup of $F$ and $F / H \cong(F / N) /(H / N)$ is a Lie group.

Corollary 1. Let $G$ be a locally compact group with closed normal subgroup $F$. If $P\left(G / G_{0}\right)$ is compact (in particular if $G / G_{0}$ is compact) then $F$ has maximal compact subgroups, any two of which are conjugate by some element of $G$, and $F$ is an $\mathscr{H}$-group.

Proof. The corollary follows immediately from Theorem 1 [2] and Proposition 2. 
Lemma 7. Let $G$ be a locally compact group with a closed compactly generated normal subgroup $F$. If $G / F$ is an $\mathscr{H}$-group and $F$ has a maximal compact normal subgroup $Q$ such that $F / Q$ is discrete, then $G$ is an $\mathscr{H}$-group.

Proof. Since $G$ is an $\mathscr{H}$-group if and only if $G / Q$ is, we can assume that $Q$ is trivial and consequently $F$ is discrete and finitely generated. Let $\varphi: G \rightarrow \mathscr{A}(F)$ be the canonical map of $G$ into the automorphism group of $F$. Let $P$ be the kernel of $\varphi$. Since $F$ is discrete and finitely generated, $\mathscr{A}(F)$ is discrete; so $P$ is an open normal subgroup of $G$. Now, let $G_{1} / F$ be the maximal compact normal subgroup of the $\mathscr{H}$-group $G / F$. If $G_{1}$ has a maximal compact normal subgroup $K$ such that $G_{1} / K$ is a Lie group, then $K$ is maximal in $G$ and $G / K$ is a Lie group. Thus, it is sufficient to prove that $G_{1}$ is an $\mathscr{H}$-group. To do this we let $M=G_{1} \cap P$. Then $M$ is an open normal subgroup of $G_{1}$ and $M / M \cap F \cong M F / F$ is compact. It follows that $M$ is a $(Z)$-group. Thus $M=B(M), P(M)$ is a characteristic open subgroup of $M$, and $P(M) \cap F=$ $e$. The last follows from the fact that $P(M)$ is central in $F$ and $F$ has no nontrivial compact normal subgroups. Since $(M \cap F) P(M) / M \cap F$ is compact so is $P(M)$ compact. These two groups are isomorphic since $P(M) \cap F=e$. Thus, $P(M)$ is the maximal compact normal subgroup of $M$ and is open in $G_{1}$ since $P(M)$ is open in $M$ which in turn is open in $G_{1}$. Consequently $G_{1} / P(M)$ is discrete. Since any compact normal subgroup of $G_{1}$ intersects $F$ in the identity, $P(M)$ is maximal compact normal in $G_{1}$. That completes the proof.

Lemma 8. If $G$ is a locally compact group with a closed connected normal subgroup $H$ such that $G / H$ is an $\mathscr{H}$-group, then $G$ is an $\mathscr{H}$-group.

Proof. If $Q / H$ is the maximal compact normal subgroup of $G / H$ with Lie factor isomorphic to $G / Q$, then $Q$ has a maximal compact normal subgroup $K$ such that $Q / K$ is Lie since $Q$ is almost connected. It follows that $K$ is maximal compact normal in $G$ and $G / K$ is Lie.

Theorem 2. If $G$ is a locally compact group with a compactly generated normal subgroup $F$ such that $G / F$ and $F / F_{0}$ are $\mathscr{H}$-groups, then $G$ is an $\mathscr{H}$-group. Proof. It follows from Lemma 7 that $G / F_{0}$ is an $\mathscr{H}$-group and from Lemma 8 that $G$ is an $\mathscr{H}$-group.

The following example shows that, if instead of assuming $F$ is normal and $G / F$ is an $\mathscr{H}$-group, we assume that $F$ is a uniform subgroup, the resulting counterpart to Theorem 2 does not hold.

Example 2. le t $B$ be a compact group with no nontrivial normal subgroups, and let $D$ be a finite group of automorphisms of $B$. Let

$$
F=\left(\sum_{-\infty}^{\infty} B_{i}\right) \times_{\eta}\left(\prod_{-\infty}^{\infty} D_{i}\right),
$$

where $B_{i}=B$ and $D_{i}=D$ and the semidirect product is defined by coordinatewise action. Now let $G=F \times{ }_{\eta} Z$ where $Z$ is the integers and the semidirect 
product is defined by shifts. Let $H=\left(\Sigma B_{i}\right) \times{ }_{\eta} Z$. The homogeneous space $G / H$ is compact, and $H$ is an $\mathscr{H}$-group, but $G$ is not an $\mathscr{H}$-group.

Another example indicates the need for $F$ to be compactly generated.

Example 3. Let $Z_{i}=Z$, the integers, and $K_{i}=Z_{2}$, the two element group. Let

$$
G=\left(\sum_{i=1}^{\infty} Z_{i}\right) \times_{\eta}\left(\prod_{i=1}^{\infty} K_{i}\right)
$$

with coordinatewise action

$$
n \rightarrow-n \text { in } Z_{i}, \quad H=\sum_{i=1}^{\infty} Z_{i}
$$

with the discrete topology, and $K=\prod_{i=1}^{\infty} K_{i}$ with the product topology. Both $H$ and $G / H$ are $\mathscr{H}$-group, but $G$ is not Lie and the maximal compact normal subgroup of $G$ is the identity. Thus $G$ is not an $\mathscr{H}$-group.

Lemma 9. If $G$ has a compact normal subgroup $K$ such that $G / K$ is a Lie group and $F$ is a closed normal subgroup of $G$ such that $P(G / F) \cap B(G / F)$ is compactly generated, then $G / F$ is an $\mathscr{H}$-group.

Proof. The hypothesis of the lemma is satisfied by $G / K$ and $F K / K$; and, if $(G / K) /(F K / K) \cong G / F K \cong(G / F) /(F K / F)$ is an $\mathscr{H}$-group, so is $G / F$. Thus, we can assume that $G$ is a Lie group. Now $P(G / F) \cap B(G / F)^{-}=H$ is a closed characteristic subgroup of $G / F$, Corollary 1.3 [15]. By Proposition 4 [15], $H$ is an $F C$-group and thus has a maximal compact normal subgroup $K$ by 3.16 [7]. It follows that $H$ is the maximal compact normal subgroup of $G / F$ as desired.

Using the above lemma we have the following immediate consequence of Theorem 2.

Theorem 3. If $G$ has a closed normal compactly generated subgroup $F$ such that $G / F$ and $F$ are $\mathscr{H}$-groups and $P\left(F / F_{0}\right) \cap B\left(F / F_{0}\right)$ is compactly generated, then $G$ is an $\mathscr{H}$-group.

Proposition 3. Let $G$ be a locally compact group, and let $F$ be a closed compactly generated normal subgroup of $G$ such that

1. $F$ and $G / F$ are $\mathscr{H}$-groups.

2. $F$ is a (CA)-group, i.e. the group of inner automorphisms is closed in the group of all automorphisms of $F$.

3. $B(F)$ is compactly generated.

Then $G$ is an $\mathscr{H}$-group. 
Proof. Let $K$ be the maximal compact normal subgroup of $F$. Since the hypotheses on $G$ and $F$ are satisfied by $G / K$ and $F / K$ [16], we can assume that $F$ is a compactly generated Lie group without nontrivial compact normal subgroup. Thus $\mathscr{A}(F)$ is a compactly generated Lie group. Since $G / F$ is an $\mathscr{H}$-group we can choose $G_{1}$ to be a closed normal subgroup of $G$ containing $F$ such that $G_{1} / F$ is compact and $G / G_{1} \cong(G / F) /\left(G_{1} / F\right)$ is a Lie group. Let $\varphi: G_{1} \rightarrow \mathscr{A}(F)$ be the canonical map, and let $M$ be the kernel of $\varphi$. Since $F$ is a $(C A)$-group, $\varphi(F)$ is closed in $\mathscr{A}(F)$. Thus $M F=\varphi^{-1}(\varphi(F))$ is closed in $G_{1}$. Since $M F / F$ is compact and $F$ is compactly generated, $M$ is $\sigma$-compact. Thus $M / M \cap F \cong M F / F$. Since $M \cap F$ is central in $F, M \cap F \subset B(F)$, hence compactly generated since $B(F)$ is a compactly generated $F C$-group. Thus $M$ is a compactly generated $(Z)$-group, $P(M)$ is the maximal compact normal subgroup of $M$, and $M / P(M)$ is a Lie group. Now $G / G_{1}$ is Lie and $G_{1} / M$ is Lie since it can be embedded in $\mathscr{A}(F)$. Finally, $G / P(M)$ is a Lie group. The proof is complete when $P(M)$ is shown to be maximal compact normal in $G$. To prove that we let $Q$ be a compact normal subgroup of $G$. Then $Q F / F \cong Q / Q \cap F$ is a compact normal subgroup of $G / F$. Thus $Q F \subset G_{1}$. Now $q f q^{-1} f^{-1} \in F \cap Q=e$ for $q \in Q$ and $f \in F$, since $F$ has no nontrivial compact normal subgroup. Thus $\varphi(q)(f)=q f q^{-1}=f$. Thus $Q \subset P(M)$, and $P(M)$ is consequently maximal in $G$.

If a locally compact group $G$ is an $\mathscr{H}$-group, the maximal compact normal subgroup is contained in $B(G)$; so $K \cap B(G)$ is trivially dense in $K$. For totally disconnected groups this density condition is sufficient to assure that $G$ is an $\mathscr{H}$-group.

Proposition 4. Let $G$ be a totally disconnected locally compact group with a torsion free discrete uniform subgroup $P$. If there is a compact open subgroup $K$ of $G$ such that $K \cap B(G)$ is dense in $K$, then $G$ is an $\mathscr{H}$-group.

Proof. Since $G / P$ is compact and $P$ is torsion free, $G$ has maximal compact open subgroups $M$, Proposition 1.9 [14]. Thus $M$ contains the closure of the subgroup generated by $\left\{g k g^{-1}: g \in G, k \in K \cap B(G)\right\}$ which contains the closure of the subgroup generated by $\left\{g k g^{-1}: g \in G, k \in K\right\}=K^{G}$. Thus $K^{G}$ is a compact open normal subgroup of $G$. The conclusion follows.

\section{ACKNOWLEDGMENT}

The second and third authors wish to express their appreciation to the University of Miami for the hospitality extended to them during their visit for the Fall of 1988.

\section{REFERENCES}

1. R. W. Bagley and T. S. Wu, Maximal compact normal subgroups and pro-Lie groups, Proc. Amer. Math. Soc. 93 (1985), 373-376.

2. R. W. Bagley and M. R. Peyrovian, A note on compact subgroups of topological groups, Bull. Austral. Math. Soc. 33 (1986), 273-278. 
3. R. W. Bagley, T. S. Wu, and J. S. Yang, Locally compact groups: maximal compact subgroups and $N$-groups, Math. Proc. Cambridge Philos. Soc. 104 (1988), 47-64.

4. __, Compactly generated subgroups and open subgroups of locally compact groups, Proc. Amer. Math. Soc. 103 (1988), 969-976.

5. __ On a class of topological groups more general than SIN groups, Pacific J. Math. 117, (2) (1985), 209-217.

6. S. Grosser and M. Moskowitz, On central topological groups, Trans. Amer. Math. Soc. 127 (1967), 317-340.

7. __ Compactness conditions in topological groups, J. Reine Angew. Math. 246 (1971), 1-40.

8. S. Helgason, Differential geometry and symmetric spaces, Academic Press, New York, 1962.

9. G. Hochschild, The structure of Lie groups, Holden-Day, 1965.

10. D. Montgomery and L. Zippin, Topological transformation groups, Interscience Publ., New York, 1955.

11. G. D. Mostow, Self-adjoint groups, Ann. of Math. 62 (1955), 44-55.

12. M. R. Peyrovian, Maximal compact normal subgroups, Proc. Amer. Math. Soc. 99 (1987), 389-394.

13. D. F. S. Robinson, $A$ course in the theory of groups, Springer-Verlag, New York, 1982.

14. S. P. Wang, Compactness properties of topological groups, Trans. Amer. Math. Soc. 154 (1971), 301-314.

15. __ Compactness properties of topological groups, II, Duke Math. J. 39 (1972), 243-251.

16. D. H. Lee and T. S. Wu, On CA topological groups, Duke J. Math. 37 (1970), 515-521.

17. T. S. Wu and Y. K. Yu, Compactness properties of topological groups, Michigan Math. J. 19 (1972), 299-313.

18. T. S. Wu, Closures of Lie subgroups and almost periodic groups, Bull. Inst. Math. Acad. Sinica 14 (4) (1986), 325-347.

Department of Mathematics, University of Miami, Coral Gables, Florida 33124

Department of Mathematics, Case Western Reserve University, Cleveland, Ohio 44106

Department of Mathematics, University of South Carolina, Columbia, South Carolina 29208 